\title{
Emplois de réputé, censé et supposé dans les définitions de dictionnaires de langue contemporains ${ }^{1}$
}

\author{
Féron, Corinne et Coltier, Danielle \\ Université du Maine \\ corinneferon1@yahoo.fr \\ coltier.danielle@,wanadoo.fr
}

\section{Introduction}

La définition de dictionnaire est à priori caractérisée par un effacement énonciatif ${ }^{2}$ : les embrayeurs en seraient exclus ${ }^{3}$, de même que certaines unités lexicales telles les adjectifs affectifs (Kerbrat-Orrechioni 2002 : 95). La présence du locuteur se manifeste pourtant dans certaines définitions, par le biais d'unités lexicales axiologiques ( $c f$. par exemple Temple 1996: 38, à propos de la définition de chinoiserie), ou d'unités modalisatrices, telles que prétendre ${ }^{4}$; dans :

(1) CLEF. PSYCHOL. Clef des songes. Ce qui permet de déchiffrer les rêves, de les expliquer, d'accéder à leur sens caché; $p$. méton., ouvrage prétendant livrer cette explication. (TLF)

prétendant indique que la proposition « un ouvrage dénommé clef des songes livre cette explication » est au moins discutable aux yeux du locuteur.

On s'intéressera ici aux emplois de trois unités modalisatrices ${ }^{5}$ dans les définitions de dictionnaires de langue contemporains : censé, supposé et réputé $e^{6}$ dans la construction : $N$ (être) censé/supposé/réputé $X$, où $N$ est un groupe nominal et $X$ un infinitif, un adjectif ou un groupe nominal.

Des études antérieures (Coltier et Dendale 2010, Féron et Coltier 2009) proposent une étude de ces unités dans le cadre de la polyphonie; notre étude s'inscrit dans un autre cadre théorique, celui des univers de croyance (Martin 1987, 1992) ${ }^{7}$.

Nous considérons que, d'une façon générale, ces unités ont une incidence modale ( $c f$. Martin 2005), en ce sens qu'elles suspendent la valeur de vérité du contenu propositionnel $p$, construit par $N X$ : en ellesmêmes, elles ne donnent pas d'indication sur l'attitude du locuteur concernant le statut de $p$ au regard du vrai et du faux. Le co-texte peut cependant donner des informations sur l'attribution par le locuteur d'une valeur de vérité ; les énoncés comprenant ces unités sont ainsi compatibles avec des suites qui indiquent que le locuteur tient le contenu pour vrai, aussi bien qu'avec des suites indiquant qu'il le tient pour faux ou douteux :

\footnotetext{
Jules est censé/supposé étudier. Et il étudie en effet. Jules est censé/supposé étudier. Mais / Et il n'étudie pas. Jules est censé/supposé étudier. Est-ce le cas ?

Cette plante est réputée guérir l'acné. En effet, elle est très efficace. / Je l'ai essayée, son effet a été rapide.

Cette plante est réputée guérir l'acné. Mais en réalité, elle est inefficace. / Je l'ai essayée, elle n'a aucun effet.

Cette plante est réputée guérir l'acné. Est-elle vraiment efficace ? / Mais est-elle efficace?
}

Ces unités ont en outre en commun de spécifier le statut de $p:$ c'est une proposition non actuelle, une vue de l'esprit; la situation que dénote $N X$ n'a pas de réalité empirique. Censé, supposé et réputé sont donc 
des modalisateurs, intra-prédicatifs, dont le rôle est de qualifier le mode d'existence de la proposition à laquelle ils s'associent, de la catégoriser.

Par ailleurs, toutes trois introduisent une image d'univers ; mais réputé se différencie assez nettement des deux autres ${ }^{8}$ : dans l'image d'univers évoquée, le contenu propositionnel $p$ est pris en charge avec la valeur vraie ; cette unité donne donc à p le statut de croyance, alors que censé et supposé ne spécifient pas en eux-mêmes la valeur de vérité de $p$ dans l'image évoquée.

Notre corpus est constitué des énoncés définitionnels tirés de trois dictionnaires informatisés (le Trésor de la langue française - compte non tenu des définitions empruntées -, le Nouveau Petit Robert et le Dictionnaire de l'Académie française DAF9), dans lesquels les unités en question portent sur un segment de la définition'. Les analyses que nous présenterons ici concernent des définitions de noms, toutes à priori paraphrastiques.

Dans cette étude, notre objectif sera double : d'une part, il s'agira de décrire comment se construit l'interprétation des unités dans les définitions de dictionnaires : nous examinerons dans un premier temps quelques définitions qui comprennent une occurrence de réputé, puis, conjointement, des définitions où figure une occurrence de censé ou de supposé; d'autre part, nous analyserons le statut des segments $N$ (être) censé/supposé/réputé $X$ dans les définitions au regard du modèle de la définition aristotélicienne et de la notion de stéréotype, et nous envisagerons l'incidence des segments en question sur la possibilité de substituer en discours la définition au mot défini.

\section{Réputé}

Nous reprenons la définition de corbeau étudiée par Fradin et Marandin (1979) :

(2) CORBEAU. ORNITH. Grand oiseau (Passereaux) au plumage noir, au bec fort et légèrement recourbé, réputé charognard. (TLF)

Cette définition fournit, parmi les «traits descriptifs des référents de corbeau », d'une part « des traits visibles » («au plumage noir...»), d'autre part un trait («charognard »), qui, de par la présence de réputé, est « présenté comme un élément de croyance » (Fradin et Marandin 1979:61-62). De fait, on interprète réputé comme équivalant à «qui a la réputation d'être»: réputé confère à $p$ («le corbeau est charognard ») le statut d'un dire exprimant une croyance collective. L'image d'univers évoquée est donc celle d'une communauté.

Le signifié de réputé inclut donc, comme le nom réputation et comme l'adjectif réputé « renommé », les traits /dire/ (réputé est donc un marqueur évidentiel, présentant l'information comme empruntée) et /croyance collective/, que le verbe réputer ( «tenir pour, considérer comme ») ne comprend pas : en ce sens, réputé est analysable comme un adjectif, sémantiquement différent du verbe réputer.

Il arrive que le co-texte fournisse des indications sur l'image d'univers dans laquelle $p$ est vrai, par exemple un adverbe comme autrefois, qui limite la vérité de $p$ à une image d'univers définie temporellement :

(3) CONSOUDE. Plante de la famille des bourraches dont une espèce (grande consoude ou consoude officinale), autrefois réputée capable de consolider les chairs, est utilisée en médecine comme astringent. (TLF)

La propriété introduite par réputé intervient également dans l'identification de l'image d'univers; dans le cas suivant : 
(4) MEDIUM Personne réputée douée du pouvoir de communiquer avec les esprits $(N P R)$

le contenu propositionnel $p$ ( « un médium est doué du pouvoir de communiquer avec les esprits ») ne peut être vrai que dans une image d'univers dans laquelle $p$ ', «il est possible de communiquer avec les esprits », et $p$ », « les esprits existent», le sont également ( $c f$. la notion de consistance des univers de croyance, Martin, $1987: 31$ ).

Lorsqu'il s'agit d'une propriété unanimement admise (il existe des animaux qui sont charognards) et en l'absence de toute indication co-textuelle, l'interprétation se construit à partir de ce qui fait la spécificité de l'énoncé définitoire : celui-ci émane d'un locuteur assimilé au lexicographe, qui, lorsqu'il rédige la définition d'un nom désignant une classe naturelle ou une fonction, un rôle social, décrit les traits permettant d'identifier ce à quoi le mot-entrée permet de référer ; lorsqu'un de ces traits est introduit par réputé, le lecteur l'interprète comme n'étant pas unanimement admis par la communauté que représente le dictionnaire.

En revanche, rien ne dit si $p$ (« le corbeau est charognard ») est vrai ou non pour le locuteur; il en est de même dans la définition de médium. Mais dans la définition de consoude, la présence de autrefois, qui renvoie à une communauté à laquelle le locuteur n'appartient pas, peut laisser supposer, pour des raisons pragmatiques, que le locuteur n'évalue pas $p$ comme vrai - qu'il le tienne pour simplement possible, indéterminé ${ }^{10}$ ou faux.

\section{Censé/supposé}

Les deux unités paraissent sémantiquement proches : dans un énoncé donné, la substitution de l'une de ces unités à l'autre entraine un changement ténu, difficile à expliciter. Nous décrirons ainsi la spécificité de chacun :

- supposé présente la proposition qu'il introduit comme une vue de l'esprit particulière : il signifie toujours que la proposition est attribuable à un être du monde et suggère l'idée d'une forme de raisonnement, à partir d'un fait admis par hypothèse ;

- censé en revanche présente la proposition comme un attribut de l'objet dénoté par $N$, comme une propriété « attendue » de l'entité à laquelle l'unité lexicale permet de référer.

Dans les énoncés comportant ces deux unités - comme dans ceux qui comprennent réputé - la valeur de vérité de $p$ est suspendue pour le locuteur. Mais dans les définitions que nous examinerons, le co-texte peut conduire à interpréter $p$ comme appartenant à un monde contrefactuel (section 3.1) ou à un monde potentiel (section 3.2), donc comme pris en charge dans l'univers du locuteur (respectivement comme faux et possible). Dans d'autres cas (section 3.3), rien ne permet de calculer la valeur de vérité de $p$ dans l'univers de croyance du locuteur, mais pour des raisons pragmatiques ou en raison du co-texte, $p$ est interprété comme vrai dans un univers de croyance.

\subsection{Groupe $1: p$ appartient à un monde contrefactuel}

(5) PERSPECTIVE. Perspective cavalière : perspective de convention (l'œil de l'observateur étant supposé situé à l'infini) permettant de montrer l'agencement des diverses parties de l'objet (NPR)

(6) PHYLACTERE BX-ARTS. Dans un tableau, une sculpture, banderole que tiennent certains personnages et qui porte les paroles qu'ils sont censés prononcer. (DAF9)

Supposé et censé ne donnent aucune indication sur la valeur de vérité de $p$, mais notre connaissance du monde nous permet de savoir que l'œil de l'observateur n'est pas placé à l'infini, que les personnages qui 
portent une banderole ne parlent pas : la proposition $p$ n'a pas de correspondant dans le « monde de ce qui est », elle appartient à un monde contrefactuel.

On a ici affaire à ce qui est admis par convention, ce qu'explicite la définition de perspective cavalière du $D A F 9$ ("perspective de convention ») : on dessine (et on regarde le dessin) comme si c'était un fait que l'œil était placé à l'infini, on lit le contenu de la banderole comme s'il s'agissait des paroles que prononcent les personnages. Ces définitions concernent des noms relevant du domaine des arts - domaine de la convention, de la représentation - et, de ce fait, les définitions de phylactère dans certains dictionnaires n'introduisent pas de modalisateur :

(7) PHYLACTERE. ARCHEOL. Banderole à extrémités enroulées portant le texte des paroles prononcées par les personnages d'une oeuvre d'art du Moyen Age et de la Renaissance. (NPR)

Censé et supposé, en (5) et (6), ont pour rôle d'expliciter l'idée de convention qui reste implicite dans la définition de phylactère que donne le NPR (elle y est impliquée par le mot oeuvre d'art qui réfère à un objet du monde qui est le lieu d'une représentation, et non au « monde de ce qui est »).

\subsection{Groupe 2 : $p$ appartient à un monde potentiel}

(8) ARGUS Publication fournissant des renseignements spécialisés et à qui rien n'est censé échapper dans son domaine. (TLF)

(9) MULETIER Chemin, sentier muletier. Chemin, sentier étroit et escarpé qui est censé n'être gravi que par les mulets. (TLF)

Ici, la propriété que dénote $X$ ne relève pas en soi de ce qui est jugé impossible. Mais de par la présence de censé, elle est présentée comme une vue de l'esprit; on l'interprète donc comme une propriété qui n'est pas nécessairement vérifiée : il existe des objets du monde nommés argus qui n'ont pas cette propriété, on peut nommer chemin muletier un sentier par où passent des animaux de bât autres que des mulets - ce que la définition du GLLF signifie par l'introduction de l'adverbial en principe :

(10) MULETIER. Chemin muletier, sentier escarpé, difficile, destiné en principe au passage des mulets. (GLLF)

Censé situe $p$ dans au moins un monde potentiel : en ce sens, il relève de la modalité aléthique.

\subsection{Groupe $3: p$ est interprété comme une croyance}

Dans les deux groupes précédents, le co-texte permet de calculer la valeur de vérité de $p$ dans l'univers de croyance du locuteur. Ce qu'il y a de commun aux définitions qui suivent, c'est que des connaissances extralinguistiques, concernant les savoirs et croyances de la communauté à laquelle appartient le rédacteur et qu'il représente (en 3.3.1 et 3.3.2.) ou le co-texte (en 3.3.3), conduisent à interpréter $p$ comme une proposition qui n'est pas admise unanimement, et, en ce sens censé et supposé apparaissent comme des marques de « distance ».

\subsubsection{L'interprétation se construit à partir de la propriété $X$}

(11) MEDIUM. OCCULT. Personne qui est censée communiquer avec le monde des esprits, en particulier pour servir d'intermédiaire entre les vivants et les morts. (DAF9)

(12) SÉLÉNITE. Être supposé habiter la Lune. (TLF)

Les propriétés «communiquer avec le monde des esprits» et « habiter la lune » ne sont pas admises unanimement dans la communauté que représente le dictionnaire, mais peuvent l'être par une partie de la 
communauté ou par une autre communauté (par exemple dans le passé : $c f$. la définition de Sélénite dans le NPR : «Habitant autrefois présumé de la Lune ») ; $p$ est, en ce sens, interprétable comme vraie ou au moins possible dans une image d'univers.

Dans le cas de Sélénite, l'existence réelle (Martin 1976 : 48-50), d'êtres ainsi dénommés est corrélée, dans le «monde de ce qui est», à la vérification de $p$ : dans l'univers où «qqn habite sur la lune est faux », « il existe des Sélénites » l'est aussi ; au contraire, dans le cas de médium, dans l'univers où « il est possible de communiquer avec les esprits » est faux, les médiums ont néanmoins une existence réelle. Cette différence tient à l'hyperonyme : dans tout univers, il existe des personnes, des êtres humains ; quelle que soit la valeur de vérité de $p$, la proposition « il existe des personnes » est vraie - tandis que être réfère à ce qui est ou est simplement imaginaire: de la valeur de $p$ dépend l'existence de l'être. L'existence réelle des Sélénites est ainsi limitée à une image d'univers.

Dans certains cas, des groupes prépositionnels figurant avant la définition permettent l'identification de la communauté qui tient $p$ pour vrai :

(13) COMBAT. Au Moy. Âge. Combat singulier. Synon. ordalie, (plus récent) duel. Combat judiciaire. Duel entre l'accusateur et l'accusé ou leurs champions, dont le vainqueur était censé être assisté de Dieu et détenir le bon droit (TLF)

(14) CHAMAN, CHAMANE Principalement en Asie centrale et septentrionale, mais aussi ailleurs dans le monde. Genre de prêtre ou de prêtresse censés entretenir des rapports privilégiés avec les puissances surnaturelles, jouant le rôle d'intermédiaires entre les hommes et les dieux, les vivants et les morts, et qui emploient ce don pour la divination, le traitement des malades, les exorcismes, ou bien parfois à des fins maléfiques. (DAF9)

Ces groupes prépositionnels fonctionnent comme expressions cadratives : elles spécifient l'univers de discours dans lequel $p$ est vrai.

En ce qui concerne la valeur que le locuteur attribue au contenu propositionnel $p$, rien ne l'explicite dans les définitions de médium et de Sélénite. Mais pour des raisons de cohérence pragmatique - parce que $p$ n'est pas vrai pour tout locuteur - censé et supposé seront lus comme des marques de réserve du locuteur vis-à-vis de la proposition. Dans le cas de combat singulier, le groupe prépositionnel renvoyant au passé permet de construire une interprétation identique à celle que nous proposons pour consoude (cf. supra, (3) : « autrefois réputée capable de consolider les chairs »).

\subsubsection{L'interprétation se construit à partir de $\mathbf{N}$}

Dans les exemples précédents (de (11) à (14)), $N$ réfère virtuellement à un existant (hypothétique dans le cas de Sélénite), et c'est la nature même de la propriété que dénote $X$ qui conduit à situer $p$ dans une image d'univers. $N$ peut aussi désigner une entité dont l'existence réelle n'est pas unanimement admise, tandis que $X$ dénote une propriété définitoire et dont l'existence est en revanche admise :

(15) INCUBE. Didact. Démon masculin censé abuser d'une femme pendant son sommeil. (NPR)

(16) INCUBE. THÉOL. Démon masculin, supposé abuser des femmes durant leur sommeil.(TLF)

Dès lors, $p$ ne peut avoir de valeur de vérité que dans un univers où la proposition « les démons existent » est vraie. 


\subsubsection{L'interprétation se construit à partir du co-texte}

Dans d'autres cas, $N$ inclut une caractérisation qui catégorise explicitement le référent virtuel du mot défini comme inexistant dans le "monde de ce qui est» (imaginaire, fabuleux, légendaire...), comme n'ayant pas d'existence réelle ; le contenu propositionnel est alors situé d'emblée dans un univers de croyance, et plus précisément dans l'univers de la fiction, qu'il s'agisse d'une propriété unanimement admise («vivre dans le massif himalayen » ou non (« présider à un phénomène naturel ou influer sur la destinée d'un individu ou d'un groupe ») :

(17) YETI. Humanoïde légendaire, surnommé aussi «l'abominable homme des neiges ", supposé vivre dans le massif himalayen. $(N P R)$

(18) ESPRIT. Être imaginaire de la légende, censé présider à un phénomène naturel ou influer sur la destinée d'un individu ou d'un groupe. (DAF9)

\section{Analyse des définitions}

\subsection{Statut des propriétés}

Dans les définitions que nous avons citées ci-dessus, les segments réputé/censé/supposé $X$ ont des statuts divers : ils fournissent l'unique propriété distinctive dans les définitions de médium (NPR et DAF9), et de Sélénite, tandis qu'ils en ajoutent une à d'autres propriétés - distinctives ou non - dans les autres cas. Nous examinerons quelques-unes des définitions citées ci-dessus selon que le segment $N$ censé/réputé/supposé fournit une propriété distinctive, ou distinctive et nécessaire.

\subsubsection{X n'est pas une propriété distinctive}

Les définitions de corbeau et de consoude (cf. supra (2) et (3)) sont stéréotypiques : réputé $X$ ajoute un trait non distinctif qui correspond à une croyance collective concernant le référent; il ne décrit pas le référent lui-même mais la façon dont il est perçu, conçu. Dans le cas de corbeau, "réputé charognard » fournit la représentation culturelle du référent dans la communauté linguistique; dans la définition de consoude en revanche, « réputé capable de consolider les chairs » est une croyance appartenant au passé et en ce sens renvoie à un stéréotype qui n'est pas celui de la communauté à laquelle appartiennent le locuteur et le lecteur: le segment comprenant réputé fournit une information d'ordre encyclopédique, susceptible d'aider le lecteur à comprendre des emplois de ces mots dans des textes anciens ou référant à des pratiques du passé.

\subsubsection{X est une propriété distinctive et nécessaire}

Dans le groupe 2 (argus et chemin muletier), les propriétés introduites par censé (respectivement : «à qui rien n'est censé échapper dans son domaine » et «qui est censé n'être gravi que par les mulets») renvoient, comme les propriétés introduites par réputé dans les définitions de corbeau et de consoude, à une représentation du référent, mais celle-ci n'est pas présentée comme une croyance ; elle correspond à une attente : en ce sens ces définitions sont stéréotypiques. Les attentes en question sont liées à la dénomination même : c'est le fait que seuls les mulets semblent emprunter certains chemins qui motive la dénomination chemin muletier; quant à l'exhaustivité attachée à la représentation de ce qu'est un argus, elle est liée à la fois aux autres sens de ce mot que le $T L F$ cite auparavant (« Homme clairvoyant qu'on n'abuse pas, qui ne s'abuse pas lui-même », « Homme jaloux, parce que la jalousie exige une surveillance continuelle », "Surveillant, espion ») et à l'étymon - plus précisément à ce qui caractérisait le personnage mythologique d'Argus : avoir cent yeux, et donc ne rien laisser échapper.

Par ailleurs, contrairement à réputé $X$ dans les définitions de corbeau et de consoude, censé $X$ est une propriété nécessaire : le segment comprenant censé $X$ est une condition d'emploi du mot ; on ne parlera 
de chemin muletier que pour un chemin dont on attend qu'il ne soit gravi que par des mulets, ou d'argus que pour une publication dont on attend qu'elle soit exhaustive.

Ce sont aussi des propriétés distinctives : le trait "qui est censé n'être gravi que par des mulets » distingue le chemin muletier par exemple du chemin de chèvres (défini sans plus dans le TLF comme un chemin «escarpé, d'accès difficile »); le trait «à qui rien n'est censé échapper» distingue l'argus d'autres publications qui fournissent des renseignements spécialisés.

En revanche, $X$ («à qui rien n'échappe » et « qui n'est gravi que par es mulets ») en lui-même n'est pas une propriété nécessaire : $X$ appartient à l'intension du nom, mais censé indique que les référents actuels n'ont pas toujours ces propriétés ; censé signale donc un écart entre ce qui est parfois (ce que l'on dénomme argus n'est pas toujours exhaustif, un chemin muletier est parfois emprunté par d'autres animaux que des mulets) et ce qui devrait être, par définition, en vertu de l'origine du mot.

Réputé $X$, censé $X$ et supposé $X$ sont également des propriétés nécessaires et distinctives dans les définitions de médium (dans le NPR, cf. (4) et dans DAF9, cf. (11)), Sélénite, esprit : par exemple, pour médium, on ne peut nommer «médium » qu'une personne dont certains pensent qu'elle est capable de communiquer avec les esprits, ou dont il est attendu qu'elle le fasse.

\subsection{Substitualité}

La présence de censé/supposé n'affecte pas les possibilités de substitution en discours de la définition au mot défini dans les cas où $p$ correspond à une convention (perspective cavalière, phylactère) ou à une attente attachée au référent du défini (argus, chemin muletier). Dans les cas où le co-texte ou des connaissances pragmatiques conduisent à interpréter $p$ comme vrai dans une image d'univers (médium, sélénite, incube, esprit, chaman, yéti, combat, singulier), la substitution semble poser problème dans des co-textes où la vérité de la proposition introduite par censé/supposé est présupposée. Par exemple, dans :

(19) J'ai rencontré un médium il y a trois ans ; il est resté longtemps silencieux, concentré sur sa communication avec les esprits, tout ce qu'il m'a dit s'est réalisé : il a parfaitement interprété ce que les esprits lui faisaient connaitre.

un médium est une personne qui communique avec le monde des esprits, ce que la définition citée sous (11), «personne qui est censée communiquer....», ne dit pas. L'unité censé, selon notre interprétation, suspend la vérité de $p$ dans l'univers du locuteur, alors que le co-texte présuppose qu'elle est vraie.

On notera que les restrictions concernant la substitualité sont résolues en l'absence de censé/supposé; ainsi pour la définition d'incube dans le Lexis :

(20) INCUBE. Sorte de démon qui abuse des femmes pendant leur sommeil. (Lexis)

De fait, dans les définitions que donnent de ce mot le NPR («Démon masculin censé abuser... »)) et le $T L F$ («Démon masculin, supposé... ») la présence du nom démon suffit à restreindre la vérité de $p$ aux univers dans lesquels la proposition «il existe des démons» est vraie. Il en est de même pour yéti ( Humanoïde légendaire ») et esprit («être imaginaire de la légende »). En revanche, dans la définition de Sélénite, c'est supposé qui est chargé d'indiquer que l'existence réelle des Sélénites n’appartient pas, aux yeux du locuteur, au «monde de ce qui est ».

La substitution semble toujours possible lorsque la définition comprend la séquence réputé $X$. Ainsi, la définition de médium dans le $N P R$ ( «Personne réputée douée du pouvoir de communiquer avec les esprits ») nous parait substituable en discours au mot défini, alors que, comme dans (11), le nom est défini par une propriété qui n'est pas présentée comme effective ; la différence entre les deux définitions tient au fait que réputé, tout en présentant $p$ comme une vue de l'esprit (comme non actuelle), lui donne le 
statut de croyance : $p$ est vrai dans une image d'univers; dans l'univers de croyance du locuteur d'un énoncé tel que (19), la proposition « un médium est réputé doué de... », qui signifie que $p$ est vrai dans une image d'univers, et la proposition « un médium est doué de... » peuvent être vraies l'une et l'autre.

Pour autant, l'introduction de réputé dans la définition de médium entraine un effet de mise à distance : réputé donne à la propriété «être doué... » le statut d'une qualité émanant d'un dire, et à partir de là le lecteur calcule que le locuteur ne tient pas la propriété pour effective.

Au contraire, « un médium est censé communiquer avec le monde des esprits... » ne donne pas en soi à $p$ le statut de croyance - ce n'est que par un calcul interprétatif que l'interprétant est conduit à penser que certains ne tiennent pas $p$ pour vrai, que $p$ est une croyance, et censé est interprété comme une marque de réserve du locuteur. De ce fait, « un médium est censé communiquer avec le monde des esprits... » et « un médium communique avec le monde des esprits » paraissent incompatibles dans un même univers de croyance.

\section{Conclusion}

Réputé, qui se différencie des autres unités en ce qu'elle donne à la proposition le statut de croyance manifeste une attitude du locuteur vis-à-vis des objets dénommés par le mot-entrée et en ce sens dénonce l'appartenance du locuteur à un ici-et-maintenant de l'énonciation et à une communauté linguistique qui a des savoirs, des valeurs et des croyances spécifiques.

Dans le cas de censé et supposé, la nature de $p$ et/ou de $N$ ou encore le co-texte peuvent conduire à un interpréter les énoncés comme manifestant également une attitude sur les référents ( $c$. supra, division 3.3.).

Concernant les jugements axiologiques, M. Temple (1996: 38) note que « on peut soutenir que les jugements de valeur associés par les locuteurs aux catégories extralinguistiques sont inscrits au niveau du sens des mots désignant ces catégories » et renvoie à C. Kerbrat-Orrechioni (2002: 89): «le trait axiologique est une propriété sémantique de certaines unités lexicales ».

De même, la croyance collective attachée aux référents de corbeau, "être charognard »", peut être considérée comme relevant du sens lexical du nom corbeau; ce n'est en revanche pas le cas de « autrefois réputé... » qui figure dans la définition de consoude, en ce sens qu'il s'agit d'une information d'ordre encyclopédique.

Il n'en va pas de même pour les définitions relevant du type décrit dans la division 4.1.2. : dans ce cas, c'est une propriété nécessaire et distinctive qui est introduite par censé, réputé ou supposé; le sens du mot est livré via une attitude/un jugement du locuteur sur le référent - ce qui peut rendre difficile la substitution de la définition au mot défini dans certains co-textes.

De telles définitions correspondent à une conception du dictionnaire comme « instrument de communication » (Marandin 1990: 285, reprenant les propos de Putman), incluant dans les définitions des attitudes sur l'existence réelle des entités que le mot-défini dénomme ou sur l'une des propriétés distinctives des référents ${ }^{11}$.

\section{Références bibliographiques}

Borillo, A. (2005). Peut-on identifier et caractériser les formes lexicales de l'aspect en français ? In Bat-Zeev Shyldkrot, H. et Le Querler, N. (éds), Les périphrases verbales, Linguisticae investigationes, supplementa 25, 83102. 
Charaudeau, P., Maingueneau, D. (éds). (2002). Dictionnaire d'analyse du discours. Paris : Le Seuil.

Coltier, D. et Dendale, P. (2010). Êtres imaginaires et dictionnaires de langue. La polyphonie dans les définitions de dictionnaires, Recherches Linguistiques, 31, 277-297.

Féron, C. et Coltier, D. (2009). Etude sémantique des unités censé/réputé/supposé + infinitif: les limites de la synonymie, Pratiques, 141-142, Questions de synonymes, 150-164.

Fradin, B. et Marandin, J.-M. (1979). Autour de la définition: de la sémantique à la lexicographie. Langue française, $43,60-83$.

Gaatone, D. (1998). Le passif en français. Bruxelles : Duculot.

Gross, M. (1975). Méthodes en syntaxe. Paris : Hermann.

Kerbrat-Orecchioni, C. (2002). L'énonciation. De la subjectivité dans le langage. Paris : A. Colin.

Lamiroy, B. et Melis L. (2005). Les copules ressemblent-elles aux auxiliaires ? In Bat-Zeev Shyldkrot, H. et Le Querler, N. (éds), Les périphrases verbales, Linguisticae investigationes, supplementa 25, 145-170.

Le Goffic, P. (1993). Grammaire de la phrase française. Paris : Hachette.

Lehmann, A. (1980). Le féminin dans le Petit Larousse illustré de 1906 à nos jours. Etude du discours des renvois. In Chevalier, J. et al. (dir.), Discours et idéologie. Paris : P.U.F, 237-275.

Marandin, J.-M. (1990). Le lexique mis à nu par ses célibataires. Stéréotype et théorie du lexique. In Chaurand, J. et Mazière, F. (dir.), La définition, Paris : Larousse.

Martin, R. (1976). Inférence, antonymie et paraphrase. Paris : Klincksieck.

Martin, R. (1987). Langage et croyance. Bruxelles/Liège : P. Mardaga.

Martin, R. (1992). Pour une logique du sens. Paris : PUF.

Martin, R. (2005), Définir la modalité, Revue de linguistique romane, 7-18.

Temple, M. (1996). Pour une sémantique des mots construits. Villeneuve d'Ascq: Presses universitaire du Septentrion.

Wilmet, M. (2003). Grammaire critique du français. Paris : Hachette.

\section{Dictionnaires}

Dictionnaire de l'Académie française, $9^{\mathrm{e}}$ éd., ATILF/Académie française (http://atilf.atilf.fr/academie9.htm) [DAF9]

Grand Larousse de la langue française, Paris : Larousse. [GLLF]

Lexis : dictionnaire de la langue française, Paris : Larousse, 1975 [Lexis]

Nouveau Petit Robert de la langue française 2007, Dictionnaires Le Robert/Sejer, 2006. [NPR]

Trésor de la langue française informatisé, ATILF (http://atilf.atilf.fr/tlf.htm). [TLF]

\footnotetext{
${ }^{1}$ Nous suivons les « rectifications de l'orthographe » de 1990.

${ }^{2}$ Cf. par exemple Lehmann (1980 : 238) : « Le sujet de l'énonciation (les auteurs du dictionnaire) s'efface ».

${ }^{3}$ «Il existe des énoncés qui ne sont pas narratifs et qui sont dépourvus d'embrayage (par exemple une définition de dictionnaire ou un proverbe) » (Charaudeau et Maingueneau $2002: 211$ ).

${ }^{4}$ Kerbrat-Orrechioni (2002 : 123, note 1) signale ce fait et cite un exemple du Petit Larousse 1948.

${ }^{5}$ Etre censé INF est rangé dans la modalité par Gaatone (1998: 81): parmi les verbes transparents, on trouve « divers verbes qu'on peut classer sous la rubrique des modaux tels que devoir (...), être censé/supposé (...)". Borillo (2005:77) adopte une position opposée : elle cite être censé parmi les "quelques verbes assez disparates, qui ne sont ni modaux, ni aspectuels : avoir beau, avoir lieu, être censé ».
} 


\footnotetext{
${ }^{6}$ Supposé est analysable comme participe passé ; réputé est selon nous tantôt participe passé, tantôt adjectif (dans le sens de «qui a la réputation de». Quant à censé, il est catégorisé comme adjectif dans les dictionnaires contemporains que nous avons consultés. Gross $(1975: 164)$ catégorise également censé, ainsi que réputé, comme adjectifs, mais note que ce sont les seuls adjectifs à admettre un complément direct. En revanche Wilmet $(2010: 568)$ et Le Goffic (1993 : 202-203) considèrent être censé comme une construction passive, et Lamiroy et Mélis (2004 : 154) comme un verbe auxiliaire.

${ }^{7}$ D'où nos choix terminologiques ; par exemple de locuteur et non d'énonciateur.

${ }^{8}$ Les dictionnaires présentent ces unités comme synonymes ; $c f$. par exemple les définitions de censé dans le TLF ( "Supposé, réputé ») et le NPR ("Qui est supposé, réputé"); de même dans le DAF9, qui introduit par ailleurs devoir dans la définition de cette unité ("Qui est supposé, présumé, réputé faire, avoir fait, devoir faire quelque chose") et le décrit ainsi comme un déontique.

${ }^{9}$ Sont donc exclus les cas où ces unités constituent une définition synonymique du mot-entrée.

${ }^{10}$ Cf. Martin 1976 : 45.

${ }^{11}$ On relève cependant des pratiques variables à l'intérieur d'un même dictionnaire ; ainsi, tandis que le NPR et le $T L F$ intègrent censé ou supposé dans la définition de incube, aucune marque n'apparait dans celles de succube :
}

SUCCUBE THÉOL. [P. oppos. à incube] Démon qui prend l'apparence d'une femme pour avoir des relations sexuelles avec un homme (TLF)

SUCCUBE Relig. chrét. Démon femelle ( $\Rightarrow$ diablesse), qui vient la nuit s'unir à un homme $(N P R)$

Par ailleurs, les mots référant à la prédiction de l'avenir sont également dépourvus de marques dans le TLF :

ASTROLOGIE. Science de certains astres (...) considérés dans leurs relations entre eux, dans leurs positions dans les signes du zodiaque; art d'interpréter ces configurations particulières à une certaine date, établies dans une carte du ciel, en vue de déterminer le caractère de quelqu'un, de prédire l'avenir $(T L F)$ 